\title{
Modifications of the exciton lifetime and internal quantum efficiency for organic light-emitting devices with a weak/strong microcavity
}

\author{
Xue-Wen Chen, Wallace C. H. Choy, ${ }^{a)}$ and C. J. Liang \\ Department of Electrical and Electronic Engineering, University of Hong Kong, Pokfulam Road, Hong \\ Kong \\ P. K. A. Wai \\ Photonics Research Centre, Department of Electronics and Information Engineering, The Hong Kong \\ Polytechnic University, Hung Hom, Kowloon, Hong Kong \\ Sailing $\mathrm{He}$ \\ Centre for Optical and Electromagnetic Research and Joint Research Centre of Photonics of the Royal \\ Institute of Technology (Sweden) and Zhejiang University, Zhejiang University, Zhijingang Campus, \\ Hangzhou 310058, People's Republic of China
}

(Received 24 September 2007; accepted 9 November 2007; published online 29 November 2007)

A comprehensive analysis is given on the modifications of the exciton lifetime and internal quantum efficiency $\left(\eta_{\text {int }}\right)$ for organic light-emitting devices (OLEDs). A linear relation is derived between the exciton lifetime and $\eta_{\text {int }}$, which is difficult to measure directly. The internal quantum efficiency can thus be estimated easily through the measurement of the exciton lifetime. The exciton lifetimes for OLEDs with weak or strong microcavity are studied experimentally and theoretically. The modification of the exciton lifetime is well explained through the microcavity effect and surface plasmon resonance. An excellent agreement between the experimental and theoretical results is achieved. (c) 2007 American Institute of Physics. [DOI: 10.1063/1.2819610]

Organic light-emitting devices (OLEDs) with various device configurations have been the subject of intensive research due to their applications in display and lighting. ${ }^{1-4}$ Considering the microcavity effect, OLEDs can be roughly categorized into two types, i.e., weak microcavity OLEDs and strong microcavity OLEDs. Conventional bottom emitting OLEDs are weak microcavity devices, while OLEDs with distributed Bragg reflectors or two metallic electrodes are considered as strong microcavity devices. Light emission properties, including the internal quantum efficiency $\left(\eta_{\text {int }}\right)$, external quantum efficiency, exciton lifetime, and angular dependence, are distinct in the two types of OLEDs due to the Purcell effect. ${ }^{5-8}$

Exciton lifetimes of emitters in planar dielectric microcavity structures, ${ }^{9}$ near a partially reflecting surface,,${ }^{10,11}$ in weak microcavity OLEDs, ${ }^{12,13}$ and above metallic gratings ${ }^{14}$ have been investigated either theoretically or experimentally. In this letter, a comprehensive analysis is given on the modifications of exciton lifetime for OLEDs with various device structures. A linear relation between the exciton lifetime and $\eta_{\text {int }}$ will be derived, which means that $\eta_{\text {int }}$ can be obtained indirectly through the measurement of exciton lifetime. In addition, we investigate both experimentally and theoretically the exciton lifetime for OLEDs with a weak or a strong microcavity.

The theoretical analysis of exciton lifetime is based on a classical approach where the emitter is considered as an electric dipole running at a fixed current and with a random orientation. ${ }^{6,15,16}$ As a consequence of Fermi's golden rule, the radiative decay rate $\Gamma_{r}(\lambda)$ at a wavelength $\lambda$ in an OLED device is modified to ${ }^{15,16}$

${ }^{\text {a) }}$ Author to whom correspondence should be addressed. FAX: (852) 25598738. Electronic mail: chchoy@eee.hku.hk and wchchoy@hkucc.hku.uk.

$$
\Gamma_{r}(\lambda)=F(\lambda) \cdot \Gamma_{0}(\lambda),
$$

where $\Gamma_{0}(\lambda)$ is the radiative decay rate in the infinite medium. Here, $F(\lambda)$, the so-called Purcell factor, is the normalized total emission power of the electric dipole with random orientation (normalized by the total power of the dipole in the infinite medium). It should be noted that here, the emission power coupled to the metal electrode is also included in $F$. We consider such decay channel as radiative decay since this part of emission can potentially be coupled out from the device through, for example, patterning the substrate. ${ }^{17} \mathrm{In}$ addition, we typically assume that the nonradiative decay rate $\Gamma_{\mathrm{nr}}$ does not change by varying the thickness of the hole-transport layer (HTL) or the electron transport layer (ETL) in the device. The initial quantum efficiency $\eta_{0}, \eta_{\text {int }}$, and exciton lifetime $\tau_{\text {OLED }}$ in the OLED are given by

$$
\begin{gathered}
\eta_{0}=\int_{\lambda_{1}}^{\lambda_{2}} \Gamma_{0}(\lambda) d r /\left[\int_{\lambda_{1}}^{\lambda_{2}} \Gamma_{0}(\lambda) d \lambda+\left(\lambda_{2}-\lambda_{1}\right) \Gamma_{\mathrm{nr}}\right] \\
\eta_{\mathrm{int}}=\int_{\lambda_{1}}^{\lambda_{2}} F(\lambda) \cdot \Gamma_{0}(\lambda) d r /\left[\int_{\lambda_{1}}^{\lambda_{2}} F(\lambda) \cdot \Gamma_{0}(\lambda) d \lambda+\left(\lambda_{2}\right.\right. \\
\left.\left.-\lambda_{1}\right) \Gamma_{\mathrm{nr}}\right] \\
\tau_{\mathrm{OLED}}=\tau_{0} /\left[\eta_{0} \int_{\lambda_{1}}^{\lambda_{2}} F(\lambda) \Gamma_{0}(\lambda) d \lambda / \int_{\lambda_{1}}^{\lambda_{2}} \Gamma_{0}(\lambda) d \lambda+1\right. \\
\left.-\eta_{0}\right]
\end{gathered}
$$

where $\left(\lambda_{1}, \lambda_{2}\right)$ is the wavelength range of the emission and $\tau_{0}$ 


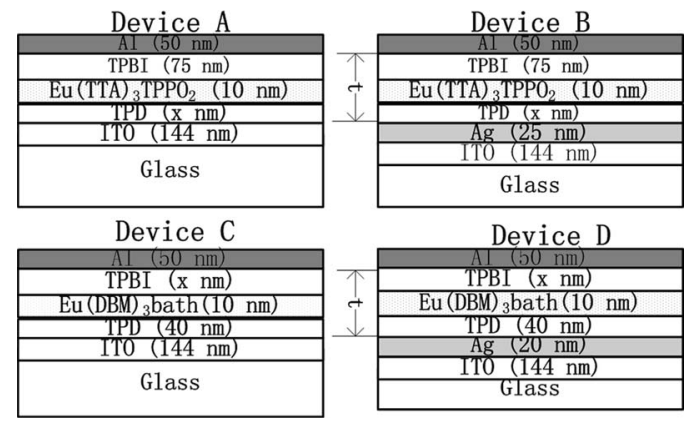

FIG. 1. Schematic diagrams of the devices A, B, C, and D.

is the exciton lifetime of the bulk emitting material. Equation (4) can be written as

$$
\tau_{\mathrm{OLED}}=\tau_{0}\left(1-\eta_{\text {int }}\right) /\left(1-\eta_{0}\right) .
$$

From Eq. (5), one observes that $\tau_{\text {OLED }}$ linearly depends on $\eta_{\text {int }}$, which means that $\eta_{\text {int }}$ can be determined from $\tau_{\text {OLED }}$. As shown above, the Purcell factor $F(\lambda)$ is a crucial quantity, which can be evaluated with the knowledge of the electric field at the location of the dipole. ${ }^{6}$ The electric field can be efficiently calculated by integrating the plane wave component of the field along a proper integration path. ${ }^{16}$

In the experiment, europium $(\mathrm{Eu})$ organometallic complexes were used in the OLEDs as the emitters. ${ }^{18}$ As shown in Fig. 1, four sets of OLEDs, i.e., devices A, B, C, and D, were fabricated to study the exciton lifetime of Eu ion based emitters in weak microcavity OLEDs (devices A and C) and strong microcavity OLEDs (devices B and D). The $\mathrm{Eu}$ complexes of $\mathrm{Eu}(a \text {-thenoyltrifluoroacetonate })_{3}$ (triphenylphosphine oxide) $)_{2} \quad \mathrm{Eu}(\mathrm{TTA})_{3}(\mathrm{TPPO})_{2} \quad$ and $\mathrm{Eu}$ (dibenzoylmethanato $)_{3}($ bathophenanthroline $) \quad \mathrm{Eu}(\mathrm{DBM})_{3}$ bath were used as the emitting layer in devices $\mathrm{A}$ and $\mathrm{B}$ and devices $\mathrm{C}$ and $\mathrm{D}$, respectively. The widely used material of bis(3-methylphenyl)-diphenyl-benzidine was used as the HTL. UV emissive (1,3,5-benzinetriyl)-tris(1-phenyl-1-Hbenzimidazole) was used as the ETL. All the materials were evaporated by thermal vacuum deposition at a pressure of $10^{-6}$ Torr. The thickness of each layer is indicated in the device structure, where $x$ denotes a variable thickness and $t$ is the total thickness of the organic layers. The transient photoluminescence (PL) was excited by pulse laser at $337 \mathrm{~nm}$.

Figure 2 shows the measured transient PL intensities of the two devices, i.e., devices A and B with the same total

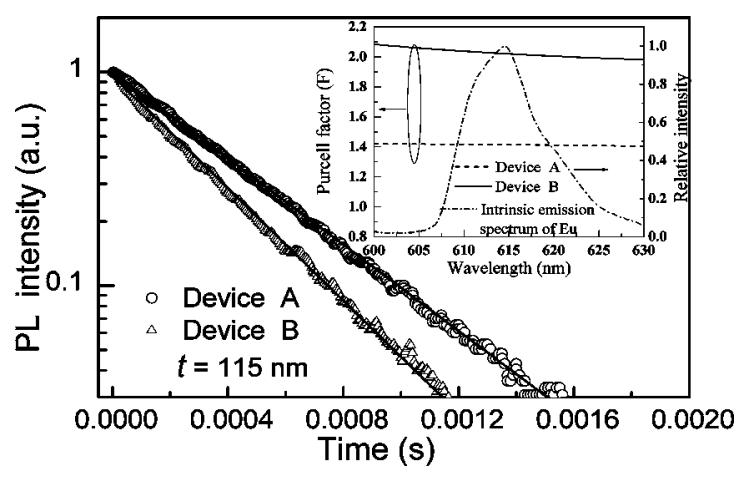

FIG. 2. The measured transient PL intensity of devices A (open circles) and B (open triangles) with the total thickness of organic layers of $115 \mathrm{~nm}$. The inset shows the Purcell factors of the two devices and the intrinsic spectrum

the exciton lifetime of device B will reach a minimum
of Eu complexes.

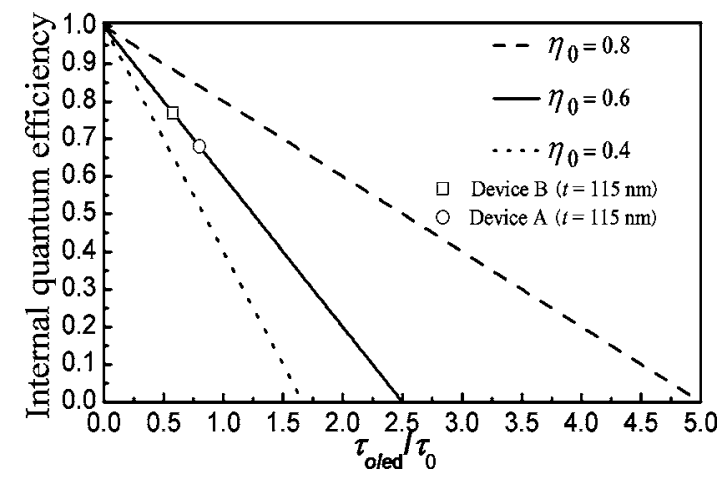

FIG. 3. Internal quantum efficiency $\eta_{\text {int }}$ varies as a function of the normalized exciton lifetime $\left(\tau_{\mathrm{OLED}} / \tau_{0}\right)$ for various $\eta_{0}$.

thickness of organic layers of $115 \mathrm{~nm}$, as open circles and triangles, respectively. The transient PL intensities with the logarithmic scales are well fitted by linear functions (shown as the solid lines), indicating an exponential decay of the intensities with time. From the linear fits, the exciton lifetimes of 0.441 and $0.325 \mathrm{~ms}$ are obtained for devices A and B, respectively. Simulations based on Eqs. (4) and (5) have been done for comparison with the experiment. The intrinsic emission spectrum of Eu complex, which is proportional to $\Gamma_{0}(\lambda)$, is shown in the inset of Fig. 2. The calculated Purcell factors $F(\lambda)$ of the two devices are also shown in the inset. According to Eq. (4) and the experimental results in Fig. 2, the initial internal quantum efficiency $\eta_{0}$ and the lifetime $\tau_{0}$ for $\mathrm{Eu}(\mathrm{TTA})_{3}(\mathrm{TPPO})_{2}$ are determined to be 0.6 and $0.55 \mathrm{~ms}$, respectively.

From Eq. (5), we observe that the internal quantum efficiency $\left(\eta_{\text {int }}\right)$ depends linearly on the normalized exciton lifetime $\left(\tau_{\mathrm{OLED}} / \tau_{0}\right)$. In Fig. 3, we plot the linear relation as dashed, solid, and dash-dotted lines for $\eta_{0}=0.8,0.6$, and 0.4, respectively. All the lines pass a fixed point $\left(\tau_{\mathrm{OLED}} / \tau_{0}=0\right.$, $\left.\eta_{\text {int }}=1\right)$ and the slopes only depend on $\eta_{0}$. Therefore, after the determination of $\eta_{0}$ and $\tau_{0}, \eta_{\text {int }}$ of an OLED device can be obtained by measuring the exciton lifetime, which is much easier as compared with the direct measurement. In Fig. 3, the open circle and square are for devices A and B $(t=115 \mathrm{~nm})$ studied in Fig. 2, respectively. Device B has a larger $\eta_{\text {int }}$ than device A due to a larger Purcell effect in device B.

Figure 4(a) shows the experimental (symbols) and theoretical (lines) exciton lifetimes of devices A and B as the functions of the total thickness of the organic layers $t$. From the figure, we observe a good agreement between the simulated and experimental results. For device A, the lifetime only changes slightly when $t$ varies since the weak cavity effect has less influence on the spontaneous emission property. However, for device B (strong microcavity OLEDs), the exciton lifetime changes significantly as $t$ varies due to the strong microcavity effect and the surface plasmon effect. In device $\mathrm{B}$, when the emissive layer is close to the silver film (small $t$ ), the emission has a good coupling to the surface plasmon modes which is absent in device A. Thus, the exciton lifetime is significantly shortened at small $t$ for device $\mathrm{B}$. As $t$ increases, the surface plasmon effect is quickly diminished and microcavity effect gradually becomes pronounced. When the resonant condition of the microcavity is satisfied, the exciton lifetime of device $\mathrm{B}$ will reach a minimum, AIP license or copyright; see http://apl.aip.org/apl/copyright.jsp 


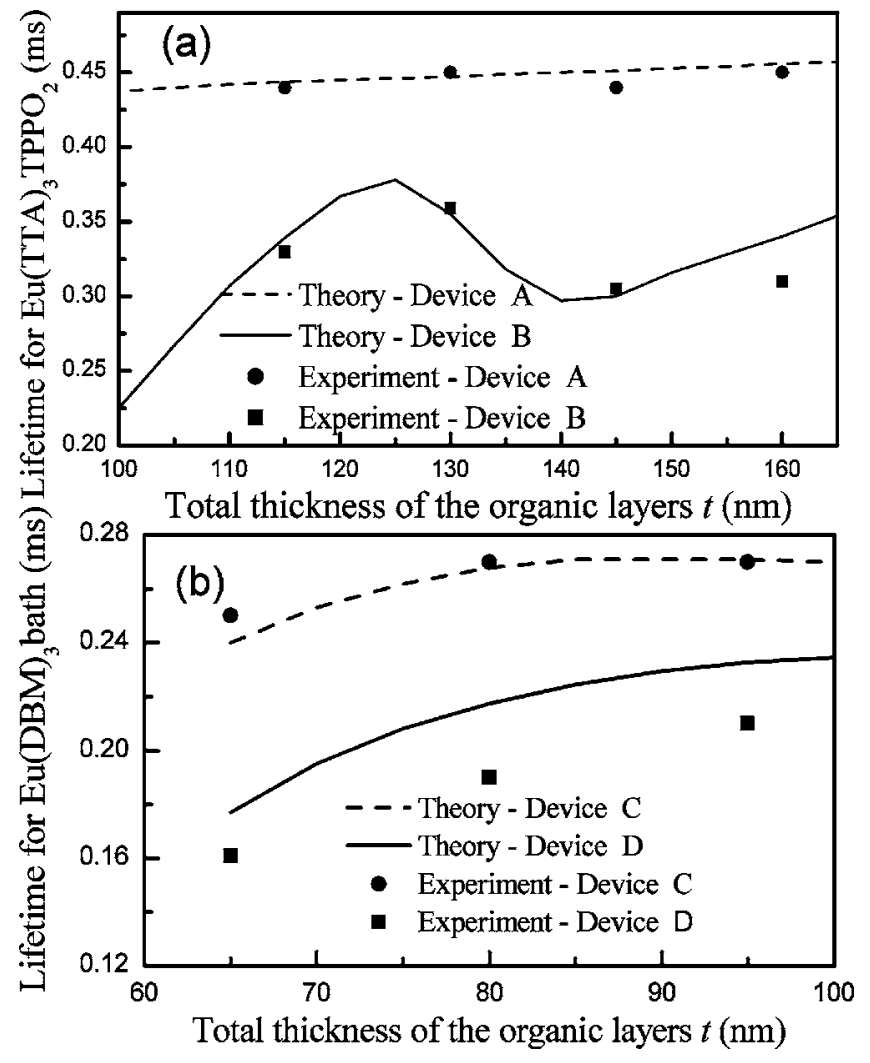

FIG. 4. Exciton lifetime as a function of total thickness of organic layers for (a) Eu(TTA $)_{3}(\text { TPPO })_{2}$ based devices A and B and (b) Eu(DBM $)_{3}$ bath based devices $\mathrm{C}$ and $\mathrm{D}$.

which indeed corresponds to the dip of the solid line at $t$ $=140 \mathrm{~nm}$ in Fig. 4(a).

Figure 4(b) shows the experimental (symbols) and theoretical (lines) exciton lifetimes of devices $C$ and $D$ as functions of $t$. Here, $\tau_{0}$ is recalibrated to be $0.33 \mathrm{~ms}$ for $\mathrm{Eu}(\mathrm{DBM})_{3}$ bath. Again, we observe a good agreement between the experimental and theoretical results. One sees that the exciton lifetimes of all the devices drop significantly as the distance from the $\mathrm{LiF}-\mathrm{Al}$ cathode becomes short. This is due to the excitation of the surface plasmon mode along the $\mathrm{LiF}-\mathrm{Al}$ cathode which exists in both types of OLEDs. Meanwhile, the microcavity effect is also pronounced for device D and, thus, the exciton lifetimes of device D are shorter. For device $\mathrm{D}$, the simulated and experimental results agree well in trend but have small discrepancy in absolute values. This may be due to some deviation in measuring the thickness of the silver film.

In conclusion, a comprehensive analysis has been given on the modifications of the exciton lifetime $\left(\tau_{\text {OLED }}\right)$ and internal quantum efficiency $\left(\eta_{\text {int }}\right)$ in OLEDs. We have shown that $\tau_{\text {OLED }}$ has a linear relation with $\eta_{\text {int }}$. This means that $\eta_{\text {int }}$, which is important but difficult for a direct measurement, can be estimated easily by measuring $\tau_{\text {OLED }}$. The modifications of exciton lifetimes in various OLEDs have been well explained through the microcavity effect and surface plasmon effect.

The authors want to acknowledge the support of UDF grant, the strategic research grant in organic optoelectronics of the University of Hong Kong, the grant (14300.324.01) from the RGC of Hong Kong SAR, China, and a grant from the National Science Foundation of China (60688401). One of the authors (P.K.A.W.) wants to thank the support from the RGC of Hong Kong SAR, China (Project No. PolyU $5226 / 03 \mathrm{E})$.

${ }^{1}$ C. W. Tang and S. A. VanSyke, Appl. Phys. Lett. 51, 913 (1987).

${ }^{2}$ V. Bulovic, G. Gu, P. E. Burrows, S. R. Forrest, and M. E. Thompson, Nature (London) 380, 29 (1996).

${ }^{3}$ L. S. Liao, K. P. Klubek, and C. W. Tang, Appl. Phys. Lett. 84, 167 (2004).

${ }^{4}$ Q. J. Sun, Y. F. Li, and Q. B. Pei, J. Disp. Technol. 3, 211 (2007).

${ }^{5}$ E. M. Purcell, Phys. Rev. 69, 681 (1946).

${ }^{6}$ W. Lukosz, Phys. Rev. B 22, 3030 (1980).

${ }^{7}$ K. Okamoto, I. Niki, A. Shvartser, Y. Narukawa, T. Mukai, and A. Scherer, Nat. Mater. 3, 601 (2004).

${ }^{8}$ X. W. Chen, W. C. H. Choy, S. He, and P. C. Chui, J. Appl. Phys. 101, 113107 (2007).

${ }^{9}$ G. Bjork, S. Machida, Y. Yamamoto, and K. Igeta, Phys. Rev. A 44, 669 (1991).

${ }^{10}$ R. R. Chance, A. Prock, and R. Silbey, J. Chem. Phys. 60, 2744 (1974).

${ }^{11}$ H. Kuhn, J. Chem. Phys. 53, 101 (1970).

${ }^{12}$ M. Kauranen, Y. Van Rompaey, J. Makij, and A. Persoons, Phys. Rev. Lett. 80, 952 (1998).

${ }^{13}$ N. Tessler, Appl. Phys. Lett. 77, 1897 (2000).

${ }^{14}$ P. Andrew and W. L. Barnes, Phys. Rev. B 64, 125405 (2001).

${ }^{15}$ K. Neyts, J. Opt. Soc. Am. A 15, 962 (1998).

${ }^{16}$ X. W. Chen, W. C. H. Choy, and S. He, J. Disp. Technol. 3, 110 (2007).

${ }^{17}$ Y. J. Lee, S. H. Kim, J. Huh, G. H. Kim, and Y. H. Lee, Appl. Phys. Lett. 82, 3779 (2003).

${ }^{18}$ C. J. Liang and W. C. H. Choy, Appl. Phys. Lett. 89, 251108 (2006). 